\title{
ESCRIBIR COSAS QUE CORRESPONDEN A LA VERDAD O SE ASEMEJAN A LA VERDAD ${ }^{1,2}$
}

Mirta Castedo ${ }^{3}$ - Adriana Bello ${ }^{4}$

\section{LOS NIÑOS Y SUS CONDICIONES ESCOLARES HABITUALES}

En este trabajo se analizan las escrituras y reescrituras de textos periodísticos realizadas por un grupo de alumnos de $5^{\circ}$ año de educación básica, en una escuela pública de zona semirrural. El grupo está integrado por veinte alumnos, trece niños y siete niñas de entre diez y doce años de edad ${ }^{5}$. Pertenecen a familias que en su mayoría se dedican a la producción hortícola, siendo sus ingresos económicos insuficientes para cubrir las necesidades básicas de vida; estos grupos familiares, calificados en "extrema pobreza", son originarios de distintas provincias del interior del país y de países limítrofes. Esta diversidad geográfica se expresa en diversidad cultural, así como en diferentes valoraciones de los actos de lectura y escritura.

Durante toda su escolaridad y hasta el momento en que se introduce la secuencia didáctica que aquí se analizará, estos niños participaron en modalidades tradicionales de enseñanza.

Se les enseñó a leer y escribir mediante el método de palabra generadora, propuesta didáctica que en este caso comienza por la enseñanza de las vocales para luego ir introduciendo, una a una, palabras que presentan las diferentes consonantes y sus combinaciones. Cada palabra se descompone en sus sílabas y letras, luego se "recompone" y se pasa a la siguiente. Es decir, no se privilegia la función de comunicación de la lengua escrita, sino que se presenta una visión fragmentada del lenguaje escrito. Se enseñan en forma gradual, según cierto grado de complejidad creciente, determinadas palabras de a una por vez. A medida que cuentan con un repertorio suficiente de palabras, con frecuencia de una misma letra, se los inicia en la copia de oraciones. Una vez aprendidas todas las letras y las combinaciones silábicas simples y compuestas, los niños deben redactar pequeñas "composiciones" escolares alejadas de los textos de uso social. Aproximadamente al llegar a tercer año, se propone la realización de actividades descontextualizadas para la ejercitación de contenidos sintácticos, morfológicos y semánticos. En síntesis, se enseña a escribir primero palabras, luego oraciones donde se aplican conocimientos gramaticales y, por último, se propone producir textos más extensos que sólo tienen sentido dentro de las escuela.

En cuanto a la lectura, se la concibe inicialmente como un mecanismo y cuando este se considera adquirido, predomina una modalidad de lectura puramente escolar, la lectura oral "para ser evaluado". La situación típica de lectura es aquella en la que todos los alumnos deben seguir atentamente un mismo texto, esperando ser nombrados por la maestra, para leer cada uno en voz alta, hasta ser interrumpido por ella en un punto elegido al azar, para que continúe otro compañero.

En el caso particular de la escuela analizada, otra característica de las prácticas habituales de enseñanza, es el escaso tiempo dedicado a la lectura y a la escritura. En parte el hecho puede obedecer a la existencia de condiciones materiales no facilitadoras (falta de libros..., revistas, materiales e instrumentos variados y atractivos para escribir, así como de lugares aptos para leer sin ser molestado por ruidos u otras distracciones...). Además, ciertas necesidades de los niños como alimentación, vacunación, cuidado bucal, documentación, etc., son frecuentemente atendidas por la escuela, hecho que resta tiempo didáctico. 
El ausentismo es alto, sobre todo por la combinación de razones climáticas con la precaria condición de salud de los niños, quienes se enferman con frecuencia. Se agrega, además, que en las condiciones de vida de las zonas rurales, la necesidad de leer y de escribir en la vida cotidiana, es relativamente más escasa que en zonas urbanas.

La lectura y escritura fueron significadas por estos y muchos otros niños como prácticas puramente escolares, cuyo aprendizaje se relacionaba directamente con la cantidad de "actividades" realizadas en el cuaderno. Se puede afirmar que a lo largo de la escolaridad, la lectura y la escritura no representaron para ellos prácticas sociales que trascendiesen los límites del aula y del cuaderno de clase.

\section{Modificación de la propuesta didáctica}

Los datos analizados en este trabajo se obtuvieron en un contexto didáctico en el que se modificaron las situaciones de enseñanza. La docente a cargo del grupo de niños, Sra. Marcela Birocho, participó durante dos años en un taller de capacitación para maestros rurales ofrecido por la Universidad Nacional de La Plata. ${ }^{6}$ Voluntariamente, motivada por el interés en encontrar alternativas de enseñanza que evitaran el fracaso reiterado de muchos alumnos y permitiesen aprendizajes de mejor calidad para todos, asistió a un taller mensual junto a otras docentes de escuelas similares y recibió la visita en su grado de un miembro del equipo del proyecto con una frecuencia semanal. Durante los talleres se discutían diferentes actividades, analizaban producciones de los niños y planificaban propuestas de trabajo para el aula. La visita tenía el propósito de ayudar a la docente a poner en práctica, en el contexto de su aula en particular, lo acordado en los talleres, es decir, atender a las dificultades que se presentaban en la tarea.

Inicialmente la docente debió sortear el desinterés de los alumnos por actividades como leer el diario, buscar información sobre determinado tema, anticipar el posible contenido de un texto, dejar registro de las actividades planificadas para la semana, etc., porque las mismas se alejaban de las propuestas tradicionales de la escuela. Era muy común escuchar la pregunta de los niños "¿Cuándo empezamos a trabajar?", refiriéndose a los acostumbrados ejercicios en sus carpetas. Pero en el transcurso del año escolar, lo novedoso, leer y escribir textos completos en circunstancias comunicativas específicas y planificar y revisar lo que se escribe, se transformó en una práctica habitual para todos los alumnos.

Durante el ciclo escolar en el que se desarrolló la secuencia que aquí se analiza, se trabajó también con elaboración de reportajes, lectura de textos de ficción siguiendo a varios autores, lectura de textos informativos, elaboración de resúmenes para estudiar y diversas actividades en torno a la biblioteca.

Se considera que el interés de este trabajo puede residir en que los textos escritos por los alumnos fueron producidos en el marco de propuestas de enseñanza distintas a las vividas durante sus historias escolares. Los datos permiten hacer evidente que una propuesta didáctica que plantee la situación de escribir textos completos de uso social para ser leídos efectivamente por otros, y que genere en los usuarios problematizaciones sobre cuestiones comunicativas, lingüísticas y cognitivas involucradas en la actividad, es factible con niños de un medio social y económico desfavorecido como el rural.

Fundamentalmente, se intentará dar cuenta de los tipos de problemas que estos niños enfrentan como escribientes de notas periodísticas, es decir, cuando tienen que "escribir cosas que corresponden a la verdad o se asemejan a la verdad", así como de los tipos de problemas que es posible que se planteen cuando revisan y reescriben estos textos. Se trata, además de aquello que es posible lograr en determinadas condiciones didácticas, en una institución cuya historia de prácticas predominantes no contempló, hasta el momento de la intervención, las ejercitaciones culturales de la escritura y los problemas que su desarrollo plantea a los usuarios.

8 - Universidad ORT Uruguay 


\section{Los textos}

Los textos que se propuso escribir a los alumnos fueron periodísticos, destinados al periódico mural de la institución y de otras escuelas conectadas entre sí a través del taller de docentes acerca del cual se comentó anteriormente.

Específicamente, los alumnos escribieron noticias: textos que informan sobre hechos recientes que interesan a un grupo de personas más amplio que aquellos que lo conocen por haber participado. Las noticias contienen las circunstancias precisas sobre el hecho informado: qué sucedió, a quién, dónde, cuándo, cómo, por qué, con qué resultados o consecuencias... El escritor se propone que su lector confíe en los datos que le proporciona, hace que los mismos resulten creíbles porque se encarga de buscar y verificar fuentes de información, las cuales cita o hace inferir al lector. Se considera una norma ética en los periódicos de circulación en nuestra cultura, que la opinión y la información no den lugar a confusión y para esto existen variados tipos de marcas e indicios ofrecidos al lector.

Las noticias deben presentarse como unidades informativas completas donde los datos precisos sobre lo acontecido funcionan como tópicos organizadores de la progresión temática. Sus partes (título, sobretítulo, copete, primer párrafo, cuerpo de la nota) exigen que se vuelva a escribir la información en forma expandida o reducida (por ej.: el título generalmente reduce la información del primer párrafo mientras que el cuerpo de la nota la amplía).

Estos textos se escriben en estilo formal, valiéndose de oraciones enunciativas generalmente breves, en tercera persona; es frecuente el uso de oraciones impersonales y de la voz pasiva.

\section{Condiciones escolares de producción de los textos}

Para los alumnos el sentido de la propuesta residía en hacerse responsables de informar a la comunidad educativa y a niños de otras escuelas sobre acontecimientos sobresalientes. El trabajo con esta secuencia didáctica se apoyó en la lectura frecuente de periódicos . Al mismo tiempo, para la escritura de esos acontecimientos, se dependía de que "sucediera algo". Los hechos sobresalientes de la comunidad cuyas escrituras se analizarán en el presente artículo fueron: una intoxicación originada en un basural y un choque.

Simultáneamente, se plantearon propósitos didácticos para la tarea: sabiendo de las dificultades que la escritura sobre un hecho real significa para los niños, -hecho en el cual se encontraban involucradosse buscó apoyar en los particulares usos y estructuras de esos textos para facilitar la conceptualización de algunos problemas. Estos propósitos fueron de varios tipos:

1. Fundamentalmente, la puesta en práctica de un proyecto de producción de notas y noticias para el periódico mural intentó crear una condición didáctica en la cual la escritura tuviese un verdadero destinatario, para quien adquiriese sentido encontrar formas lingüísticas adecuadas para comunicarse.

2. Este tipo de textos, como otras narraciones, daría oportunidades a los niños para elaborar problemas de la producción de la escritura como la elección del léxico, el uso de la puntuación, la combinación de distintos tiempos verbales, el empleo de referencias, etc.

Pero además, particularmente en estas notas, se plantearían algunas posibilidades de aprendizajes específicos. La escritura de las notas brindaba oportunidades para:

a) Discutir y resolver problemas relativos a cómo dar a conocer hechos distinguiendo la información sobre lo sucedido de la opinión, la crítica o la advertencia basadas en dicho suceso. 
b) Encontrar las formas para dar veracidad y credibilidad al escrito, buscando, verificando y citando fuentes.

c) Producir un escrito donde adquiriese sentido usar y mantener un registro de lengua formal, muy diferente al habla cotidiana de estos niños, el cual, al ser leído por otros (externos al grado), requeriría claridad y corrección en su diagramación, caligrafía y ortografía.

3. Al escribir sobre hechos que comprometían culturalmente a los autores, la tarea generó también la reflexión sobre sus propias condiciones de vida; es decir, el escribir se constituyó, al mismo tiempo, como un medio de reflexión sobre el lenguaje y sobre el mundo que el lenguaje toma como objeto.

\section{Procedimientos de transcripción y análisis}

En todos los casos, las escrituras iniciales, siempre realizadas en equipos de dos a cuatro alumnos fueron producidas en nivel alfabético, "aquel en el cual los niños han comprendido la existencia de una correspondencia entre sonidos elementales del habla y letras, pero aún no dominan estas correspondencias sobre el plano ortográfico convencional" (Ferreiro, E.; Pontecorvo, C.; Ribeiro Moreira, N.; García Hidalgo, I.- 1996).

Las escrituras fueron analizadas desde sus versiones normalizadas; es decir, transcriptas $\sin$ errores de ortografía, pero sin ningún agregado de puntuación ni otro tipo de elementos.

Además, se transcribieron estas versiones en enunciados o cláusulas, "definidos operativamente como una unidad organizada que tienen como centro un verbo conjugado" (Ferreiro y otros, ob. cit.). Las mismas autoras, "por razones prácticas", deciden incluir "los turnos de habla en discurso directo" como "una unidad tipo enunciado". Se adoptó esta forma de transcripción porque permitió analizar con mayor claridad aspectos de la organización lingüística del escrito.

Con el propósito de facilitar su lectura, las producciones de los niños se presentarán distribuidas en tales enunciados que, obviamente, no coinciden con las líneas gráficas del texto.

\section{SECUENCIA DE TRABAJO EN EL AULA Y ANÁLISIS DE LAS PRODUCCIONES ESCRITAS OBTENIDAS DURANTE SU DESARROLLO}

\subsection{Primera secuencia didáctica}

\section{Situaciones didácticas}

Se trabajó intensamente la lectura y comentario cotidiano de periódicos, incluyendo lectura en voz alta de la docente, y exploración y lectura de los niños en equipos. La intervención de la docente se orientó a tematizar sobre qué se puede encontrar escrito en los periódicos, en qué tipo de periódicos, en qué parte.

El interés de los niños se centró en la lectura de notas policiales y deportivas. La primera y principal dificultad residió en la distinción entre diferentes tipos de textos incluidos en el periódico, no sólo entre diferentes textos periodísticos (como nota, noticia, editorial, opinión...) sino también entre textos con funciones muy diferentes como noticia y propaganda. En efecto, ante intervenciones de la docente como "¿En qué parte del diario podemos encontrar lo que pasó ayer?", los niños señalaban tanto la foto de un choque como la propaganda de un supermercado o un aviso clasificado...

10 - Universidad ORT Uruguay 
Es decir, ante la pregunta planteada en situación de contrato didáctico buscaban todas las respuestas a su disposición, demostrando la escasa o nula frecuentación del periódico.

La docente insistía: “¿Nos enteramos de algo que pasó en el aviso del supermercado?”... y los niños buscaban una respuesta: "Sí, porque nos enteramos de las cosas que están baratas".

Ante estas dificultades, la docente no lograba organizar discusiones sobre la veracidad de los hechos, la distinción entre información y opinión, la actualidad de los contenidos, etc.

Mientras se desarrollan estas situaciones sucedió el primer hecho que dio lugar a la producción de una nota ( "Los jugos vencidos").

El hecho: un camión arrojó jugos vencidos en un basural a cielo abierto próximo a la escuela. Algunos niños, alumnos del establecimiento, recogieron los jugos y los llevaron a sus hogares. Allí los prepararon e ingirieron y, como consecuencia, varios se intoxicaron.

\section{Escrituras analizadas.}

17 piezas de escritura; 14 primeras escrituras producidas por 8 equipos de alumnos, dado que 6 equipos realizaron una primera versión que desecharon para luego escribir una segunda versión (que no es reescritura de la anterior sino nueva producción); 3 producciones presentan una versión reescrita.

\section{Análisis de las escrituras ${ }^{8}$}

\section{Primeras escrituras}

Las 14 primeras escrituras contienen 153 enunciados; la más breve, 3 y la más extensa, 25.

La característica fundamental de estas notas es su neto carácter apelativo: 134 enunciados pueden caracterizarse como consejos, pedidos y órdenes, aun cuando se expresan en tercera persona y con verbos impersonales propios del género periodístico; estos enunciados pueden, implícitamente, contener información, pero la misma es de carácter general ("te agarrás vómitos, diarreas y otro tipo de enfermedades..."), es decir, no referida al suceso específico.

Sólo 19 enunciados, distribuidos en 4 de las 14 producciones, contienen información precisa sobre el suceso relatado (qué, quiénes, cuándo, dónde, cómo, por qué, para qué...), siempre combinados con los enunciados del primer tipo.

Es decir, 10 producciones son de carácter predominantemente apelativo (como la que ejemplifica el texto 1) mientras que el resto combina la apelación y la información (ejemplificada por el texto 2). Por cierto, ambas producciones muestran las numerosas dificultades tanto ortográficas como léxicogramaticales de estos textos que, por momentos, hacen muy difícil su comprensión para el lector. 


\begin{tabular}{|c|c|}
\hline Texto $1^{9}$ & Texto 2 \\
\hline 1. La cosa vencida hacen mucho mal & 1. Los alimentos bencidos tienen una fecha \\
\hline 2. les costará la vida & 2. avia bencido \\
\hline 3. agara las cosa vencida, & 3. y una bes se bence \\
\hline 4. les agarra fermedades como diarrea comita & 4. ya no se puede comer \\
\hline 5. hy otras enfermedades & 5. y si lo comes \\
\hline 6. no hay que tiren cosa vencida & 6. te puede costar la vida \\
\hline 7. por que hay mucho chiquita & $\begin{array}{l}\text { 7. y te agaran bomitos diarreas iotros tipos } \\
\text { de enfermedades como el epatiti como el } \\
\text { colera y la diarrea }\end{array}$ \\
\hline 8. que lo garan & 8. Son cosas muy ionteresante para todos \\
\hline 9. y se lo seva a su casa. & 9. porque ay algunos \\
\hline 10.Y selo da a los chico & 10. que no tienen conciencia \\
\hline 11.y de puede sen ferman. & 11. de lo que pueden pasar les a los demas \\
\hline & 12. algunos no se preo cupan \\
\hline & 13. porque no son sus yijos \\
\hline & 14. los que se enferman \\
\hline & 15. y uno de esos es el jugo bencido \\
\hline & 16. aca cerca ay un basural \\
\hline & 17. y tiran cosas bencidas como los jugos \\
\hline & 18. que tiraron ace poco \\
\hline & 19. y los chicos llebaron a sus casas \\
\hline & 20. y quien sabe lo que le pudo aber pasado \\
\hline & $\begin{array}{l}\text { 21. Nos piden nos tomar ni comer cosas } \\
\text { bencidas }\end{array}$ \\
\hline & $\begin{array}{l}\text { 22. a cada niño le cuesta veinte minutos de su } \\
\text { vida }\end{array}$ \\
\hline & $\begin{array}{l}\text { 23. según lo afirmó el de los jugos o el de las } \\
\text { comidas vencidas }\end{array}$ \\
\hline & $\begin{array}{l}\text { 24. y de su salud en manos de la provincia de } \\
\text { Buenos Aires. }\end{array}$ \\
\hline
\end{tabular}

Los enunciados 15 a 19 del texto 2 son los únicos que contienen información específica sobre el suceso a informar y la dificultad lingüística para expresar estas ideas oscurece aún más su comunicación. 
En estos textos se pueden encontrar órdenes como "No andes en basureros..." o "Cuídese!!!....", consejos como "...Es para que los otros sepan que esos jugos están vencidos...” o "...las cosas vencidas que compran tienen que fijarse...", advertencias como “...los jugos vencidos le harán mal a la panza..." y pedidos como "...También le pedimos que no tomen ni coman cosas vencidas...".

Otra de las característica de estas primeras producciones es la yuxtaposición de expresiones leídas en los periódicos y copiadas textualmente en las notas, sin ninguna relación con el hecho relatado. Los enunciados 22 a 24 del texto 2 son un ejemplo de este tipo de yuxtaposición: los mismos fueron tomados de una nota donde el Ministro de Salud Pública de la Provincia de Buenos Aires advertía sobre los riesgos de vida para los fumadores. La única relación que estas frases tienen con el hecho a relatar es que ambas se refieren a un problema de salud.

\section{Reescrituras}

Las reescrituras se suscitan luego del intercambio de producciones entre autores, luego de lecturas y comentarios entre los mismos. En varias ocasiones es la misma docente quien lee las producciones a los niños para someterlas a propuestas de modificación. El resultado fueron tres producciones modificadas. Los alumnos consideraron que el resto era confuso pero no encontraban formas para mejorarlas. Todas las modificaciones realizadas fueron las siguientes:

\begin{tabular}{|c|c|}
\hline Autores & Enunciados reescritos ${ }^{10}$ \\
\hline \multirow[t]{2}{*}{ 1. Mariela y Gisela } & $\begin{array}{l}\text { 1. Los alimentos vencidos tienen una fecha de } \\
\text { vencimiento }\end{array}$ \\
\hline & $\begin{array}{l}\text { 2. según lo afirmó el de los jugos o el de las comidas } \\
\text { vencidas y de su salud en manos de todos los que } \\
\text { tiran basura }\end{array}$ \\
\hline \multirow[t]{4}{*}{ 2. Cecilia, María y Verónica } & 3. que por tomar sos jugos se pueden morir \\
\hline & $\begin{array}{l}\text { 4. En las últimas jornadas: La gente está comiendo y } \\
\text { tomando cosas vencidas }\end{array}$ \\
\hline & $\begin{array}{l}\text { 5. Hay gente que no tiene conciencia toman y comen } \\
\text { las cosas vencidas }\end{array}$ \\
\hline & 6. CUIDADO LA VIDA ESTÁ EN PELIGRO! \\
\hline \multirow[t]{2}{*}{ 3. Romina y Verónica } & $\begin{array}{l}\text { 7. usted se puede morir por los tabacos y el alcohol. } \\
\text { y los alimentos vencidos pueden causar que a Ud. lo } \\
\text { internen. }\end{array}$ \\
\hline & 8. que los comidas vencidos no se pueden comer \\
\hline
\end{tabular}

Las modificaciones no aportan grandes diferencias al sentido global de las notas: en la $1^{\circ}$ reescritura se corrige una agramaticalidad, en la $2^{\circ}$ se ajusta una frase copiada del periódico al contexto de la nota que se está elaborando, en la $3^{\circ}$ se reemplaza el verbo conjugado en presente con sentido atemporal por una frase verbal que le infiere carácter de probabilidad, en la $4^{\circ}$ se explicita un objeto directo eludido, en la $5^{\circ}$ se introduce una modificación en la concordancia, en la $6^{\circ}$ el pedido se transforma en advertencia, en la $7^{\circ}$ algunas micromodificaciones no evitan la yuxtaposición del "tabaco" y el "alcohol" (no pertinentes en este caso) aunque especifican las consecuencias de la ingestión de los jugos vencidos, en la $8^{\circ}$ se introduce una variación en el léxico... 
En todos los casos se trata de micromodificaciones que no alteran el sentido general de los escritos y, por lo tanto, no logran hacer de estos textos notas periodísticas que informen sobre un suceso específico y sus consecuencias para los afectados.

\section{2 Segunda secuencia didáctica}

\section{Situaciones didácticas}

Concluida esta tarea, el grupo evaluó que las notas no se podían publicar porque "no se entienden", "no se entiende lo que pasó". Pero de todos modos no encontraban otras formas para comunicar lo sucedido. La docente decidió no forzar una publicación que los niños, con buen criterio, consideraron inadecuada y les propuso seguir trabajando sin apuros, esperando tener algo mejor escrito para publicar.

De hecho, la "espera" propuesta por la docente no fue pasiva, sino que, analizadas las dificultades de las producciones de los niños, decidió desarrollar dos tipos de situaciones:

A) Relativas a la lectura de periódicos. Continuó la lectura y comentario cotidiano de periódicos, incluyendo su lectura en voz alta así como la exploración y lectura de los niños en equipos.

Las intervenciones didácticas siguieron orientándose a tematizar sobre qué se puede encontrar escrito en los periódicos (concepto de "hecho noticiable"). Pero al mismo tiempo se introdujeron intervenciones del tipo de "...el que lee este artículo, de qué se va a enterar sobre este hecho... podrá encontrar información sobre qué pasó... sobre dónde pasó.... sobre cuándo pasó... sobre a quiénes les pasó... etc.”, es decir, se intentó centrar a los niños no sólo sobre el tipo de hechos sino también sobre qué de los hechos se cuenta en la nota periodística. Otras intervenciones apuntaban a la conceptualización sobre opinión e información: “...¿Esto que dice aquí (cita) es lo que piensa el accidentado, el periodista o los testigos?... ¿Dónde dice algo acerca de lo que piensa el periodista sobre este hecho?...”. -Se introdujeron, además, reflexiones sobre las formas de enunciar de las notas periodísticas, fundamentalmente a través de contrastes como los siguientes: "Para informar que unos chicos se intoxicaron gravemente con los jugos vencidos, ¿cómo aparecerá esto escrito en el diario?: 'Chicos, cuídense, comer cosas vencidas los pueden matar' o 'Un grupo de niños que comió alimentos vencidos fue internado en el Hospital Interzonal, encontrándose en grave estado'...”.

B) Lecturas no periodísticas (divulgación científica y textos escolares) relativas a los contenidos involucrados en las notas: los basurales, la recolección de residuos, las razones y consecuencias del vencimiento de alimentos, las normativas gubernamentales al respecto y las distintas responsabilidades institucionales. Este tipo lecturas se encontraba motivado por el interés sobre el tema que el hecho suscitó, pero didácticamente cumplía, al mismo tiempo, un propósito importante: crear condiciones para que los niños pudieran interpretar mejor los textos leídos y producir los suyos con mayor conocimiento sobre la temática en la cual el suceso se contextualizaba. El conocimiento sobre el tema permitiría informar y opinar sobre el suceso con un mejor nivel de argumentación.

Mientras tanto se generaba una dificultad didáctica: el hecho perdía actualidad y, por lo tanto, dejaba de ser noticia... En esa circunstancia se produjo otro hecho susceptible de ser informado.

El hecho: al salir de la escuela conduciendo un automóvil, una mujer embistió a un motociclista que circulaba por la avenida sobre la cual se encuentra el establecimiento. Este hombre fue herido y hospitalizado, aunque sin gravedad. 


\section{Escrituras analizadas}

22 piezas de escritura; 6 primeras escrituras producidas por 6 equipos de alumnos, todas las producciones presentan entre 3 y 4 versiones reescritas.

\section{Análisis de las escrituras}

\section{Primeras escrituras}

Las 6 primeras escrituras contienen 56 enunciados; todos las producciones presentan entre 8 y 11. Este primer dato, sobre la poca variación en la extensión de los escritos, contrasta con la marcada discrepancia con respecto de la primera producción. Se podría sostener que el grupo de alumnos tenía una idea más estabilizada y compartida acerca de qué cantidad de elementos hay que enunciar en una nota como ésta.

Más allá de la extensión, todos los enunciados informan, con menor o mayor grado de precisión y adecuación al lenguaje periodístico, sobre el hecho ocurrido. Sólo dos enunciados, que desaparecen en las reescrituras, tienen carácter apelativo.

En todas la producciones se enuncia qué sucedió ( “...hubo un choque...", “...se chocaron...” o “...sucedió un choque...”), quiénes chocaron (“...los accidentados son Mónica Sánchez y Gerardo Tizo...” o ..."la señora se llama Mónica Sánchez y el señor se llama Gerardo Tizo”.). En 4 de las 6 producciones aparecen expresiones tales como "El lunes pasado..." o "El día tres de junio..." (cuándo) y "la calle es 13 y 86 " o "en la escuela n ${ }^{\circ} 72$ " (dónde).

Si bien en todas las producciones se alude a cómo sucedieron los hechos y cuál es el estado actual de las víctimas es allí donde se registran las mayores variaciones en las expresiones lingüísticas para comunicarlo. En algunos casos aparecen expresiones muy coloquiales como "ahora el hombre está bien y la mujer sufre una gran tristeza" o "y entonces la moto venía el auto arrancó y se chocaron...". De todos modos, estos enunciados, a diferencia de la producción sobre los jugos vencidos, informan sobre el hecho específico; la dificultad que subsiste es la combinación de un lenguaje coloquial con uno periodístico.

La transcripción de los dos textos siguientes ejemplifica los "extremos" en la adecuación de las escrituras al estilo periodístico. En el texto 3 es donde aparecen mayor cantidad de expresiones coloquiales y en el 4 se presenta la mayor aproximación al lenguaje periodístico. 


\begin{tabular}{|c|c|}
\hline Texto 3 & Texto 4 \\
\hline 1. El lunes $3 / 5$ la señora miró para la isquierda & 1. Un choque y un accidente. \\
\hline 2. y no miró para la derecha & $\begin{array}{l}\text { 2. El día } 3 \text { de junio sucedió un choque en la } \\
\text { escuela } \mathrm{N}^{\circ} 72\end{array}$ \\
\hline 3. entonces la moto venia & $\begin{array}{l}\text { 3. resulta que un auto conducido por una } \\
\text { señora de nombre Mónica sanches }\end{array}$ \\
\hline 4. el auto arranco & 4. atropelló al Señor Gerardo Tizo \\
\hline 5. y se chocaro & 5. todo sucedió \\
\hline 6. y el hombre está erido & $\begin{array}{l}\text { 6. cuando Monica Sanchez trajo a su hija al } \\
\text { colegio }\end{array}$ \\
\hline 7. la señora se llama Monica Sanchez & 7. y luego salio sin mirar \\
\hline 8. y el señor se llama gerardo Tiso & 8. y justo en ese momento venia Gerardo tizo \\
\hline 9. la calle es 13 y 86 & 9. y lo atropello. \\
\hline 10. esta muy erido & $\begin{array}{l}\text { 10. El hombre haora esta internado en el } \\
\text { Hospital Sanmartín. }\end{array}$ \\
\hline 11. y haora esta en el hospital san Martín & \\
\hline
\end{tabular}

El texto 3 comienza relatando, a la manera de una narración cotidiana, el hecho sucedido (enunciados 1 a 6), luego especifica la identidad de los accidentados, el lugar del hecho y las consecuencias. En el texto 4, luego del título, se anuncia en sólo 3 enunciados ( $n^{\circ} 2$ a 4 ) cuándo sucedió qué, dónde, cómo y entre quiénes; una expresión típicamente periodística ("todo sucedió cuando") introduce una vuelta sobre los hechos donde se aportan mayores detalles, incluyendo las consecuencias de lo sucedido.

\section{Reescrituras}

Como puede anticiparse desde la descripción de las escrituras analizadas, una diferencia notable es la cantidad de veces que se reescribe y el hecho de que todas las escrituras fueron reescritas. Este dato de por sí denota una actitud diferente ante la producción, pero además, supone mayor competencia para producir y probar modificaciones en los textos. En efecto, en las escrituras de los jugos vencidos los alumnos encontraban que sus escritos "no quedaban como noticias", pero no hallaban alternativas para mejorarlas. En estas escrituras la situación es distinta: los niños vuelven una y otra vez sobre los textos intentando mejorarlos y es la maestra quien decide concluir las revisiones a fin de que el hecho no pierda actualidad.

¿Qué tipo de modificaciones se realizan? . Se registran agregados de información sobre el momento en que sucedió la acción (por ejemplo: "el día 3 de junio" se transforma en "el día 3 de junio a las 12:30"), sobre el lugar de los hechos (ej; "en la escuela n n $^{\circ}$ " pasa a ser "en la puerta de la escuela $\mathrm{n}^{\mathrm{o}} 72$ " y "en el hospital", "en el hospital San Martín") y sobre la circunstancia en que ocurrieron los hechos (ej. "atropelló al señor Gerardo Tizo" se transforma en "atropelló al señor Gerardo Tizo que venía en una moto") . Se agregan, también, en sucesivas versiones, copetes y sobretítulos tales como "Un herido de gravedad (sobretítulo), Choque en la puerta de la escuela. (Título), Chocó un auto contra una moto (copete)". Se registran sólo dos supresiones, ambas en caso de redundancia (ej: "un choque y un accidente" se reemplaza por "un choque"). Se constatan varias sustituciones lexicales que, o bien hacen

16 - Universidad ORT Uruguay 
pensar en la búsqueda de un léxico específico relativo al tipo de texto producido ( ej:"hubo un choque" por "se produjo un choque" o "el hombre" por "el herido") o bien intentan evitar reiteraciones de raíces de palabras que provocarían cacofonías ( ej: "y salió del colegio y no miro y entonces en ese momento venía una moto conducida por Gerardo Tizo" se transforma en "y salió del colegio conduciendo su automóvil y no miró y entonces en ese momento venía una moto manejada por Gerardo Tizo").

Es decir, por un lado se encuentran los agregados de títulos, sobretítulos y copetes que son particularmente interesantes por el hecho de que suponen volver a escribir una y otra vez el mismo contenido expandiéndolo o reduciéndolo. Todas las reescrituras evidenciaron este problema que supone un tipo de trabajo con la lengua escrita que para estos niños se plantea con sentido por primera vez en su historia escolar: es el tipo de texto el que exige volver a escribir lo que se acaba de escribir y según en qué sector de su estructura se está elaborando, se tratará de expandir o de reducir. El resto de las modificaciones, la mayoría, son siempre microestructurales, afectan el interior de los enunciados o, a lo sumo, la relación entre enunciados contiguos. En estos últimos casos, la preocupación está depositada en las formas retóricas propias del lenguaje periodístico.

Por otra parte, la tarea de revisión, no se reduce a las modificaciones observables en la escritura. Dos pequeños pasajes de un registro de clase muestran una gama de reflexiones (que no siempre desembocan en modificaciones efectivas del escrito) y que ilustran algunas de las preocupaciones y problemas de los niños al escribir.
1.Gisela
(Releyendo el copete) Chocó
2.Cecilia
(Dicta corrigiendo) Chocaron
3.Gisela No es que chocaron muchas veces...
4.Cecilia Chocaron es que chocó un auto con una moto.
5.Gisela No entiendo.
6.Cecilia La señora acá (señala un punto en la mesa) y el hombre acá (señala otro punto) es que chocaron un auto con una moto, nena!!! Dos chocaron...

7.Gisella Ah!!! Y el auto iba conducido solo, nena!!!

8.Cecilia (Dictando) Un auto conducido por Mónica Sánchez....

9. Gisela (Releyendo) La señora no se dio cuenta y chocaron, entonces sufrió heridas graves

10. Cecilia ¿Quién? La señora no sufrió heridas graves...
11. María
El hombre es...

12 Gisela El conductor... (dirigiéndose a la maestra) ¿ así se dice?

13. (La docente no escucha el pedido de Gisela)

14. Cecilia Sí, dale. Si ella es la conductora él es el conductor.

15. María (Releyendo) Cuando dobló...

16 Cecilia Cuando dobló no, al doblar (enfatizando la alternativa propuesta )

17 Gisela (Dictando el texto modificado) Al doblar (enfatizando) la señora no se dio cuenta y chocaron, entonces el conductor (enfatizando) sufrió heridas graves

18. Cecilia (a María) Sí, dale, poné ... 
Las expresiones "chocó" y "chocaron" pueden ser igualmente aceptables dependiendo del contexto en que se las ubique; el problema no parece pasar inadvertido para las niñas gracias a que Gisela seguramente está pensando en "chocó un auto contra una moto" y Cecilia en "chocaron un auto y una moto". Lo interesante es que Gisela interpreta el "chocaron" como "muchos choques" y su incomprensión provoca la explicitación de su compañera. Cuando Gisela comprende la idea de Cecilia la acepta, pero entonces la ausencia de la explicitación del conductor promueve un nuevo reclamo para su par. Está claro que para Gisela la expresión "chocaron un auto y una moto" representa, desde el sentido, un auto que chocó solo... aunque desde el punto de vista del lector adulto se infiere la presencia de un conductor. Es decir, la preocupación por incluir la identidad de la conductora del auto no proviene de la necesidad de agregar más información específica a la noticia, sino de una posición típica de los niños cuando comienzan a revisar sus escritos: explicitar todo, como si el lector no colaborara con el escritor infiriendo lo no dicho.

En el segundo pasaje se plantea, por un lado, el problema de la elisión del sujeto que "sufrió heridas graves", dado que la última persona nombrada es "la señora", Cecilia advierte la inadecuación. María y Gisela demuestran comprender inmediatamente el problema planteado por su compañera y entonces buscan el vocablo más adecuado para el contexto. La docente que en ese instante no está a disposición de las niñas, no responde y es nuevamente Cecilia quien decide la discusión, desde los saberes de los que dispone y pone a disposición de sus pares: ya han utilizado la palabra "conductora", por lo tanto el masculino será "conductor". El cambio de "cuando dobló" por "al doblar" seguramente obedece a que la expresión con infinitivo otorga a la frase un carácter más impersonal y, por lo tanto, más próximo al registro periodístico. Nada en el intercambio observable entre estas niñas indicaría que se trata de un conocimiento explícito sobre el lenguaje, sino simplemente de un saber sobre el lenguaje que se está usando.

Más allá de la importancia relativa de los cambios realizados y de la corrección y adecuación de los mismos, la actitud de las niñas al revisar muestra varias cuestiones. Por una parte, se manifiesta algo que podría ser definido como un compromiso del escritor con su texto, hecho importante en el contexto del aula: esta claro que las niñas no están sólo corrigiendo para cumplir con una tarea escolar sino también para que su texto se transforme en una pieza de escritura publicable. Pero además, a diferencia de lo que ocurrió en la primera revisión, no sólo encuentran inadecuaciones sino que hallan algunas estrategias para resolver los problemas que se les plantean.

En síntesis, los tipos de contenidos sobre los que es necesario informar están presentes desde la primera versión de las notas del choque. Apartándose del consejo, el pedido o la advertencia, los enunciados informan, y lo hacen con un principio de adecuación al género periodístico. Todavía, la explicitación de las razones de los cambios en el proceso de revisión es muy incipiente; se trata más bien de modificaciones por como "se usa" escribir en una noticia que por como "se sabe" que se escribe.... pero el hecho no impide que estos textos sí resulten publicables.

\subsection{Tercera secuencia didáctica}

\section{Situaciones didácticas}

Concluida la nota sobre el choque, los alumnos, incentivados por la conciencia de haber avanzado en sus conocimientos sobre la escritura de estos textos, "reclamaron" volver sobre el problema del basural. Si bien el hecho ya no constituía una noticia, dado que había perdido actualidad, no dejaba de ser un episodio publicable en virtud de que los niños de las otras escuelas lo desconocían. Ya no se trataría de una noticia sino de una nota periodística sobre el basural.

No se vuelve a revisar las primeras notas, sino que se inician nuevas producciones. Pero los saberes y vivencias con los que se cuenta para comenzar la escritura ya son muy diferentes.

18 - Universidad ORT Uruguay 
A partir de la escritura y revisión de las notas del choque se poseen otras experiencias sobre las formas retóricas para expresar un hecho en lenguaje periodístico. Pero además, también han discutido sobre el concepto de fuente de la información y credibilidad de las fuentes. En efecto, habiendo observado cierta tendencia de los alumnos a "inventar" datos para completar las notas cuando no los poseían, las intervenciones de la docente se orientaron a hacer más explícito un aspecto inobservable en el producto periodístico: de dónde se obtiene la información. Se analizaron distintos tipos de fuentes, incluidos los estudios de un tema que realiza un periodista cuando tiene que informar sobre unos hechos que no comprende totalmente. Se vincula este estudio sobre el tema que realiza el periodista con el trabajo de lectura y discusión que los alumnos realizaron sobre basurales, alimentos vencidos, normas municipales.

Es decir, se vuelve a escribir, pero con muchos más saberes tanto sobre la forma de hacerlo como sobre el contenido.

Un dato importante en el análisis de estas producciones es que se explicita un conflicto entre la valoración del hecho por los niños y sus familias y la apreciación que del mismo tienen los docentes. En todas estas discusiones estuvo presente la distinción entre diferentes actos de habla: cuándo y cómo se informa, cuándo y cómo se opina, se argumenta, se advierte, se persuade..., cuáles son los actos que se incluyen en un periódico, en qué parte, bajo que condiciones...

El punto de mayor conflicto se plantea en estos términos. Por un lado, los niños y sus familias pertenecen a sectores sociales de extrema pobreza. Para muchos, es una práctica habitual acudir al basural para recolectar elementos para reutilizar o vender (inclusive, para comer). Para ellos, "tener cuidado" es "no lastimarse" mientras se busca algo para recoger. Para las docentes, "tener cuidado" es no ir al basural, dando por sobreentendido que es una práctica perjudicial para la salud de los niños. Sin duda, esto es objetivamente así, pero plantearlo en el aula suponía desautorizar los consejos y valoraciones de las familias. Sólo después de varias clases y discusiones los docentes tomaron conciencia que algunas aparentes incomprensiones del material leído y algunas expresiones de los escritos, obedecían a que los niños partían de una valoración diferente del hecho, al principio confusa para los adultos que conducían el grupo.

En la escritura, muchas condiciones no dichas o dichas ambiguamente parecían obedecer a faltas de competencias léxicogramaticales. Sin embargo, el problema era en realidad más complejo: ciertos hechos eran difíciles de sostener públicamente por los autores ya que eran ellos mismos quienes serían "censurados" por los actos de los cuales participaban.

\section{Escrituras analizadas}

14 piezas de escritura, 7 primeras escrituras de las cuales 2 producciones presentan 2 reescrituras, 3 presentan 1, y 2 no tienen ninguna reescritura (son desechadas y los alumnos se suman a trabajar en otros equipos). Por lo tanto, existen 5 producciones finales.

\section{Análisis de las escrituras}

\section{Primeras escrituras}

Las 7 primeras versiones contienen entre 7 y 21 enunciados. Desde su primera lectura se observa que el grado de dificultad para escribir sobre este hecho no es equivalente al del choque. Tanto la claridad de las ideas expresadas como los recursos lexicogramaticales parecen, en muchos casos, "retroceder". 
Son mayores también las discrepancias en calidad entre las producciones. El hecho a relatar es, sin duda, más complejo, pero además es más conflictivo dado que despierta valoraciones diferentes en el grupo que comenzaron a explicitarse y se instalaron en la discusión como consecuencia de escribir sobre el mismo.

Las dos escrituras menos claras y precisas son las de los dos grupos que desechan su producción. Una de ellas es la siguiente:

\begin{tabular}{|ll|}
\hline $\begin{array}{l}\text { Texto } \mathrm{n}^{0} 5 \\
1 .\end{array}$ & Los jugos bencidos $\#^{11}$ (título) \\
2. & Jugos vensidos que alguna gente tiran \\
3. & y no se da cuenta \\
4. & qe puede hacer mal a muchas personas.\# (copete) \\
5. & El caso de los jugos vencidos ocurrió el 13 de abril en un basural \\
6. & y la hora hera a las 12:30 h \\
\hline
\end{tabular}

Tal vez lo más significativo es la ausencia de la explicitación del hecho en sí mismo ya que en el copete se hace referencia, pero de manera imprecisa. Sin embargo, aún esta nota no puede compararse con las primeras producciones: la advertencia del copete se completa con una información precisa sobre el momento, hay marcas propias del género como "el caso de" u "ocurrió" y, sobre todo, hay un intento de que el tema aludido se explicite cuando se pasa del título al copete y de éste al incipiente cuerpo de la nota: "jugos vencidos" aparece en el título y en el primer enunciado del copete, el cuerpo de la nota comienza por "el caso de los jugos vencidos".

En el resto de las producciones se da cuenta con precisión de qué sucedió, cuándo, dónde y quiénes fueron los afectados. Como en el caso anterior, las mayores variaciones se presentan en el cómo se explica el hecho.

Los titulares son construcciones impersonales como "se acumula basura en 137 y 84 " o construcciones nominales como "jugos vencidos en la calle 137 y 84 ”. Sólo una producción presenta un sobretítulo, "Basural cerca de una escuela", donde claramente se logra anticipar un hecho que luego será caracterizado y definido a lo largo de la nota.

Cuatro de las cinco producciones presentan copetes. En tres casos hacen referencia al qué sucedió, cuándo y dónde (ej: "El día trece de abril muchos hombres vinieron a tirar muchos jugos vencidos cerca de una escuela y los chicos se los llevaron a sus casas para tomarlos."). En un caso el copete tiene un carácter más apelativo: "la gente tira basura y no tiene conciencia de lo que le puede pasar a los demás".

Todas las producciones retoman en el primer párrafo y en el resto de la nota los temas ya abiertos, los cuales se amplían aportando mayor cantidad de datos; es decir, los textos muestran que los niños saben usar una estructura periodística para organizar sus escritos. El siguiente texto puede ejemplificar el tipo de producción aludida: 


\section{Texto 6}

1. Jugos bencido (título)

2. El día 13 de Abril unos hombres vinieron a tirar muchos jugo vencidos cerca de una escuela

3. y los chicos se los llevaron a sus casas para tomarlos (copete)

4. En un basural 137 esquina 84 se encontraron muchos jugos vensidos cerca de una escuela

5. los chicos ivan y se llebában a sus casa para tomarlo,

6. los chicos de la escuela salían para sus casas

7. y vieron los jugos

8. y se lo llebavan a sus casas

9. nadie sabe quien los tiró

En esta producción se informa sobre el hecho, aunque sin aludir a sus consecuencias. Se comunica el lugar, la fecha, los participantes y aparecen datos sobre cómo sucedieron los hechos. Como en el caso anterior, tanto en el título, como en el copete y en el desarrollo se plantea el tema ("jugos vencidos"), el cual es expandido con mayores detalles en cada una de las partes. Otras marcas propias del género también están presentes: la $3^{\circ}$ persona (particularmente compleja porque "los chicos" aludidos son ellos mismos), las frases enunciativas, las expresiones impersonales ("se encontraron muchos jugos vencidos"). El último enunciado, "nadie sabe quién los tiró", muestra el uso de una forma retórica empleada por el escritor para aludir a una información que no se posee con exactitud; es un avance importante desde las primeras notas donde los alumnos se permitían "inventar" datos para cumplir con la tarea y lo es, no sólo por la conciencia sobre el tipo de quehacer del periodista, sino también porque encuentran una expresión lingüística que puede comunicar el desconocimiento de un periodista, sin mentir a los lectores, pero señalando que ese protagonista desconocido de los hechos tiene un lugar en la información.

\section{Reescrituras}

Las reescrituras se desarrollan a partir del intercambio de producciones entre autores, su lectura y comentario y, en dos casos, luego de la transcripción de los textos en el pizarrón para ser analizados por todo el grupo.

Las cinco producciones reescritas contienen 65 enunciados de los cuales 47 presentan modificaciones. al revisar:

Algunos ejemplos permiten identificar diferentes tipos de problemas que los niños se plantean
1. En el texto original.
En el texto reescrito
2. En el texto original. En el texto reescrito
algunos chicos bebieron los jugos y se intoxicaron

esto ocurrió cuando los chicos de la escuela 72 iban a la escuela 
Como en estos casos, es frecuente que las modificaciones consistan en agregados que completan datos no aportados durante la primera textualización.

\section{En el texto original.}

...el hombre tiró residuo vencido en el basural...
En el texto reescrito

... alguien tiró botellas de jugo vencido en el basural...

La expresión "el hombre" intenta mencionar un sujeto de quien se desconoce la identidad, pero al utilizar el artículo determinado, los niños advierten que el lector pensaría en "un hombre conocido" . Al no poder dar cuenta de la identidad del sujeto proponen "alguien" como alternativa que resuelve la mención del nombre propio. La sustitución de "residuos' por "botellas de jugo" busca especificar el hecho, justifican el cambio diciendo "residuo siempre tiran, puede ser cualquier cosa..."
4. En el texto original.
En el texto reescrito
El $13 / 4 / 96 \ldots$
El día 13 de abril de $1996 .$.

En este caso, un niño advierte que la forma de escribir la fecha (con barras) no la ha visto nunca en los diarios. Recurre a los periódicos y lo comprueba. Buscan algunas fechas escritas en cuerpos de notas e "imitan" las formas de hacerlo.

\section{En el texto original.}

....una camioneta tiraba jugos vencidos...

\section{En el texto reescrito}

.... se encontraron jugos vencidos...

Nuevamente aparece la discusión sobre quién tiró los jugos vencidos. En este caso, el problema no recae sobre la identidad de la persona sino sobre el tipo de vehículo (no saben si se trata de camión, camioneta u otro...). El uso de la expresión impersonal, "se encontraron jugos vencidos", resuelve este conflicto.

\section{En el texto original.}

......los chicos iban y se llevaban a sus casas para tomarlo, los chicos de la escuela salían para ir y vieron los jugos y se los llevaban a sus casas.......

\section{En el texto reescrito}

..... Los chicos iban al lugar y se los llevaban a sus casas para tomarlos. Los alumnos de la escuela $\mathrm{N}^{\circ} 72$ salían para ir a sus casas y vieron los jugos y se los llevaron ........

En este pasaje coinciden una serie de micromodificaciones.

Los primeros dos agregados ("al lugar... los") son deícticos que parecen tener la intención de evitar ambigüedades. En el contexto no son estrictamente necesarios, ya que tanto la referencia al lugar como a los objetos queda sobrentendida por los enunciados anteriores; sin embargo, esta conducta que consiste en agregar para que todo quede explícito es muy frecuente en los niños (un sejemplo similar se cita en la página 10). Si bien se manifiesta cierta falta de control sobre aquello que puede ser inferido por el lector, es importante que el agregado se realice a través de deícticos que evidencian una competencia específica. 
El agregado de la "s" final en "tomarlos" es un tipo de modificación habitual en estas producciones. Los escritos iniciales tienen numerosas omisiones de $\mathrm{s}$ y $\mathrm{n}$ finales que corresponden a marcas de plural, tanto en pronombres como en verbos, sustantivos y adjetivos ( tomaro por tomaron, lo por los [ tanto antepuesto como pospuesto al verbo], cosa vencida por cosas vencidas ..) . En ocasiones, este tipo de error parece vincularse con la variedad dialectal del habla de los escribientes, y en otras, con la pérdida de control sobre la concordancia, dado que el vocablo se refiere a un sujeto u objeto explicitado en algún enunciado "lejano". Cualquiera sea el motivo, en numerosas oportunidades estas marcas de plural se completan durante la revisión; aparentemente no se trata de una falta de conocimiento sobre la estructura de la lengua sino de un saber que no se pone inmediatamente en uso durante la primera textualización. Ésta está normalmente sobrecargada de exigencias para el escribiente, quien, sin embargo posee saberes que hace jugar al volver sobre su producción. Puede entonces controlar mejor cuestiones microestructurales ya que ha resuelto el qué escribir y con qué expresiones.

Un caso parecido de pérdida de control durante la primera textualización es "salían a sus casas", expresión aparentemente agramatical o sin sentido que en realidad fue un intento por escribir "salían para sus casas". Es muy frecuente que, como en este caso, durante la revisión se restituya la intención original del escribiente.

Las dos modificaciones restantes constituyen problemas diferentes. El reemplazo de chicos por alumnos evita una repetición no advertida en la primera textualización, a través de una simple sustitución lexical. El reemplazo de llevaban por llevaron marca que el hecho relatado corresponde a una acción puntual y no durativa. Ambos saberes tienen en común ser saberes en uso, aunque en el primer caso los niños pueden argumentar mínimamente sobre las razones del cambio ("chicos de vuelta no, pone alumnos"), en el segundo caso solo se trata de un cambio efectuado sin verbalización alguna.

La última modificación, supresión de la circunstancia a sus casas, se origina en la repetición de la construcción (presente en el enunciado anterior). Es importante señalar que los niños aceptan la posibilidad de un simple elisión. Releen, tachan, vuelven a releer y dicen " sí, se entiende...". Es decir, utilizan una estrategia opuesta a las primeras modificaciones de este pasaje, no reclaman la necesidad de sustituir para dejar explícito, aceptando en los hechos, aun sin conciencia sobre la estrategia utilizada, que el lector inferirá lo no dicho. Para un escribiente, contar con un lector que colabora en la construcción del sentido del texto, es un saber sobre su quehacer que no está presente en producciones anteriores.

Como es esperable, no todos los niños se plantean el mismo tipo de problemas. El proceso de la siguiente producción puede ilustrar el conjunto de cuestiones suscitadas en un equipo. Este ejemplo muestra no solo la forma de resolver problemas léxico- gramaticales, sino también cómo las competencias de los escribientes se ponen al servicio de comunicar aquello que se ha conceptualizado sobre el hecho. 


\section{Primera versión.}

1. Basural cerca de una escuela.\#

(espacio)

2. Se acumula basura en 137 y 84 .\#

(espacio)

3. La gente tira basura

4. y no tiene conciencia

5. de lo que le puede pasar a los demás.\#

(espacio)

6. El 13 de abril se encontrarón jugos vencidos en 137 y 84 en la Localidad de La Plata.\#

7. Todo comenzó

8. cuando los chicos de la escuela $\mathrm{N}^{\mathrm{o}} 72$ turno mañana se encontrarón con cajas de jugos. vencidos

9. los chicos del turno de la mañana cuando se llevarón para la casa

10. se llevaron jugos para tomar

11. pero ellos no sabián que eran vencidos. \#

12. Los chicos de $5^{\circ} \mathrm{B}$ de la tarde investigarón sobre el tema de la basura y los productos vencidos. \#

13. Se llegó a la conclución

14. que la gente en este momento junta basura

15. y se la lleva a la casa

16. y ace muchas cosas.

En esta nota , luego del sobretítulo (enunciado 1) y el título (2), el copete (3-5) cumple una función apelativa, advirtiendo sobre las consecuencias del hecho para la población. Pero a diferencia de las notas iniciales esta advertencia se sostiene como consecuencia de un hecho del que sí se informa con precisión.

Los datos sobre qué sucedió, cuándo, dónde, cómo y a quién, que se anuncian en el título y sobretítulo, se resuelven entre los enunciados 3 a 11. Los dos últimos párrafos (12 -16) incluyen la investigación realizada en el aula, presentando lo hecho con las conclusiones obtenidas, expresados con varias marcas de lenguaje periodístico ( $3^{\circ}$ persona, impersonal...).

La expresión “...que la gente en este momento junta basura y se la lleva a la casa y hace muchas cosas" logra expresar lo que verdaderamente fue el acuerdo del grupo sin mencionar los aspectos que ocasionaron conflicto. “...En este momento... “ alude a una discusión donde se planteó que buscar cosas en los basurales no era un hecho natural, no lo hacen siempre todas las personas, sino que constituía una circunstancia provocada por las necesidades no satisfechas de estos hogares en el momento actual. “...Hace muchas cosas..." evita la mención, muy discutida entre pares, de que estos elementos podían inclusive servir de alimentos. 
Segunda versión. (Se transcriben sólo los pasajes reescritos).

2. Se tiran jugos vencidos en 137 y 84 .

8.los chicos de la escuela $\mathrm{N}^{\mathrm{o}} 72$ turno mañana se encontrarón con cajas de jugos. vencidos,

9. los chicos del turno de la mañana cuando se hiban para la casa

10. se llevaron jugos para tomar

La segunda versión, reescrita al día siguiente por los mismos autores presenta sólo dos modificaciones. En el título se mantiene la enunciación impersonal, pero se reemplaza el hecho general "Se acumula basura..." por el específico "Se tiran jugos vencidos...". En el enunciado 9, la sustitución del "iban" por "llevaron" otorga mayor coherencia a la secuencia de acciones presentada y resuelve la reiteración de la palabra "llevaron" que aparece en el siguiente enunciado.

En la $3^{\circ}$ versión, producto de la revisión colectiva del texto, presenta mayor cantidad y variedad de modificaciones.

\footnotetext{
Tercera versión.

1. Basural cerca de una escuela.\#

(espacio)

2. Se tiran jugos vencidos en 137 y $84 . \#$

(espacio)
}

\section{La gente tira basura}

4. y no tiene conciencia

5. de lo que le puede pasar a los demás.\#

(espacio)

6. El 13 de abril se encontraron jugos vencidos en 137 y 84 en la localidad de La Plata. \#

7. Todo comenzó cuando 1 os chicos de la escuela $\mathrm{N}^{0} 72$ turno mañana se encontraron con cajas de jugos vencidos,

8. cuando se iban para la casa

9. se llevaron jugos para tomar,

10. pero ellos no sabían que estaban vencidos. \#

11. Los chicos de $5^{\circ} \mathrm{B}$ de la tarde investigaron sobre el tema de la basura y los productos vencidos. \#

12. Se llegó a la conclusión

13. que la gente en este momento junta basura,

14. se la lleva a la casa

15. y hace muchas cosas.

16. La utilizan para vender

17. y en ciertos momentos cuando no tienen para comer

18. la usan como alimento. 
En el párrafo comprendido entre los enunciados 7 y 10 se introducen modificaciones que no cambian el sentido del texto, pero mejoran aspectos lexicogramaticales. Al introducir la expresión "Todo comenzó cuando... " (7) se anticipa al lector que se va a retomar el hecho ya mencionado para narrar el episodio con mayores detalles. La supresión de "...los chicos del turno mañana..." (8), aunque omite información que precisa más la identidad de los sujetos, elimina la repetición de “...los chicos...". La sustitución de "eran" por "estaban" es el producto de una intensa discusión donde se planteó que el "estar" vencidos era un estado de los jugos en ese momento, no una cualidad permanente, es decir, discusión sobre la diferenciación semántica entre ser y estar.

Un carácter totalmente distinto posee el agregado del párrafo final (16-18). No afecta aspectos lexicogramaticales sino que amplía las conclusiones de la investigación. Lo hace en el punto más conflictivo del problema: ¿para qué se usan las cosas que se recogen en el basural?. El enunciado 17, “...y en ciertos momentos cuando no tienen para comer..." expresa la toma de conciencia sobre la circunstancia vivida. Más allá de los errores e inadecuaciones que el texto aún posee, la escritura y revisión obligó a volver a pensar sobre el hecho, volver a discutir, a reflexionar y coordinar opiniones sobre un suceso difícil de objetivar cuando forma parte de la vida cotidiana de los mismos autores. Hecho que, además, no eligieron vivir sino que les fue inevitable sobrellevar en sus condiciones de vida. La escritura generó que no sólo lo vivieran sino que también lo pudieran pensar entre todos. Se volverá sobre este concepto en las conclusiones.

Como en el caso del choque, unos breves pasajes de registro de clase muestran las discusiones que sustentan las transformaciones del texto.

DOCENTE

María alude a la credibilidad del dato en función de la verificación de su fuente.

¿Podemos poner en una nota lo que escuchamos decir?

Carlos: Podemos poner que fue un vehículo...

Nelson: Y capaz que era uno que tiró (se refiere a una persona sin vehículo)

Carlos: ¿Qué? Son un montón!! (Se refiere a que las botellas son muchas para ser trasladadas por una persona sin vehículo).

Martín: Un camión, seño, un camión 
La docente devuelve el problema al grupo, formulándolo en los términos en que se le plantearía a un periodista. Carlos lo soluciona rápidamente empleando un hiperónimo que evita la precisión sobre el tipo de vehículo. No es tenido en cuenta por sus compañeros que comienzan a hipotetizar otras posibilidades ( no para escribir, sino sobre como pudieron haber sucedido los hechos). Obviamente, solamente Carlos comprendió el problema que María señaló y Cecilia hizo propio.

Esperen, nuestro problema no era

si camión o camioneta. Nuestro problema era

que Cecilia dice que escuchó, no que vio...

Romina: Tiene que averiguar, seño, preguntar...

Verónica: Preguntar para saber bien...

Sí, pero ya pasó bastante tiempo y la gente tiene muchas versiones, unos dicen unas cosas, otros otras... ¿cómo hacemos con esto?. ¿Qué hacen los periodistas cuando les pasa esto?

A ver, hay alguna manera de escribir para que se vea que lo que estamos diciendo no lo vimos ni nadie lo vio muy bien sino que lo escuchamos... que podría ser... que no estamos seguros....

María: Sí, en el diario dice así a veces... (se refiere a notas leídas) .

Cecilia: ... Yo no me acuerdo...

Mónica (dictando): Escuchamos un comentario..

Gisela (dictando y corrigiendo la enunciación de su compañera): Se escuchó un comentario que una camioneta... 
La docente vuelve a formular la cuestión en otros términos. La respuesta de dos niñas muestran que se han ubicado en el problema planteado y proponen una solución general, compartida en otras clases, pero no aplicable al caso. La docente señala esta inadecuación y no obtiene ninguna respuesta. Reformula el problema centrándolo sobre la forma de enunciar lo sucedido bajo la circunstancia de no poseer la fuente precisa.

Lentamente comienza a coordinarse el requerimiento de la tarea con la experiencia adquirida durante la lectura, se intenta una enunciación en primera persona del plural, inmediatamente una compañera la transforma en impersonal.

Puede ser así?
Varios: Sí, porque es lo que pasó...

.....es que se escuchó...

.... que no lo vio, que lo escuchó....

(continúan todos hablando a la vez)

La docente no valida de inmediato la propuesta sino que solicita opiniones a fin de verificar cuál es el sentido para el resto de los niños. En general, comprueba que la clase está compartiendo el sentido de quienes han propuesto la solución al problema y entonces la valida

Bien, esa puede ser una solución. Lo que

decía Carlos ¿puede ser otra solución? Poner un

vehículo que puede ser camión, camioneta u otra cosa....

Puede entonces retomar la primera propuesta de Carlos para evaluarla como propuesta de solución alternativa.

Comparando la calidad y variedad de modificaciones operados durante estas reescrituras, se puede comprobar que son más numerosas y variadas que las introducidas en las notas sobre el choque. Además las discusiones dan lugar al desarrollo de más y mejores argumentaciones.

¿Desde dónde se genera esta posibilidad creciente de reflexión sobre la textualización? Por un lado, se sabe más sobre el contenido que se está comunicando, no solo en términos de meras informaciones sino que se han discutido puntos de vista y opiniones sobre el hecho y, en ese contexto, se ha discutido sobre las propias condiciones de vida. Al mismo tiempo, se han incrementado las competencias lexicogramaticales, a través de la lectura y reflexión permanente del periódico y la necesidad de reactualizar lo leído para resolver problemas de escribientes. Didácticamente, se ha introducido un factor crucial para poder avanzar en el saber: confiar en la posibilidad de los sujetos para realizar las tareas, es decir, los alumnos tuvieron "éxito" en tareas anteriores y simultáneas y los adultos les hicieron saber de diversas maneras que sus producciones eran interesantes, comunicables, legibles...

28 - Universidad ORT Uruguay 
Son los mismos niños que al iniciar la secuencia no distinguían una propaganda de una noticia. Transcurrieron pocos meses. Pero a diferencia de los años anteriores de escolaridad, durante estos meses la situaciones didácticas se centraron en el uso de la escritura y la reflexión sobre su uso.

\section{DISCUSIÓN}

Los datos aquí presentados muestran un recorte de una microevolución de un grupo escolar con una historia particular, en unas condiciones determinadas. No se reseña todo el trabajo del grupo en el área de lengua sino sólo el relativo a una secuencia didáctica en un período comprendido entre unos pocos meses (aproximadamente mayo a octubre). Como se señaló, la historia de este grupo no se caracterizaba, hasta el ciclo escolar que aquí se describe, por una verdadera práctica de escritura con sentido para los niños, sino por los típicos ejercicios escolares descontextualizados y por una escasa frecuentación de materiales escritos de circulación social. Por otra parte, se trata de un grupo de niños en "extrema pobreza" y, como es habitual en estos casos, niños con una reiterada historia de fracaso escolar y de escasa calidad de sus prácticas educativas. Al mismo tiempo, esta modalidad de trabajo didáctico es la primera experiencia para la docente que conduce el grupo.

Es en estas condiciones que pueden ser interpretados los datos expuestos, los cuales provocan algunas reflexiones.

En principio, las primeras producciones escritas muestran el precario dominio de la escritura de este grupo de niños. Tanto en su competencia sobre el sistema de representación mismo como en los conocimientos lexicogramaticales necesarios para expresar algunas ideas con una mínima claridad. Evidencian también un rudimentario conocimiento de las prácticas de escritura, donde la planificación y revisión del escrito están totalmente ausentes, así como las relaciones entre la escritura y otros quehaceres como la lectura, la discusión, la consulta de fuentes, etc.; es decir, una ausencia de prácticas propias de cultura letrada.

Más allá de hasta qué punto se transforman estos saberes, el dato que parece ser más significativo parece ser que, justamente, se transforman. A pesar de la historia personal y escolar de estos niños, se comprueba que su producción escrita se modifica en una dirección deseable: escriben más, más adecuadamente y con mayor nivel de reflexión sobre la práctica que desarrollan. Sin duda, los textos finales están mejor escritos, dado que más allá de todos los errores e inadecuaciones lingüísticas que aún poseen, llegan a ser comprensibles para cualquier lector. Al mismo tiempo, el nivel de reflexión sobre la escritura queda evidenciado en las revisiones, que pasan de estar casi ausentes en el inicio a formar parte integrante de la escritura misma en el final. Es interesante observar que a medida que evoluciona la textualizacion se complejiza la revisión del escrito y la reflexión que la misma genera..

Cabe preguntarse cuáles fueron las condiciones para que esto sucediera.

En principio, una condición parece ser tan simple que suele perderse de vista: escribir. Ciertamente, una escuela que sólo se ocupa de escribir ejercicios descontextualizados, no da oportunidades para escribir textos completos en circunstancias variadas de comunicación para ser efectivamente leídos por otros. Si los niños no tienen estas oportunidades, no pueden enfrentarse a los problemas que la escritura plantea y por lo tanto no pueden elaborar los saberes que tal práctica genera. Es decir, no pueden aprender a escribir.

Pero tampoco se trata sólo de permitir escribir. La docente sostiene una intervención que por un lado permite el uso de la escritura y, por otro, ayuda a tomar distancia de la tarea, a autoevaluarla, a reflexionar sobre ese uso. Para ello, acerca a los niños los medios necesarios para resolver los problemas que se les plantean. Se trata de buscar un punto en el cual no se resuelva por ellos, pero tampoco se los abandone a sus propias posibilidades, porque las mismas no son suficientes para poder avanzar en sus saberes. 
La evaluación permanente de las escrituras de los niños y la decisión sobre el tipo de situaciones y de intervenciones a desarrollar en función de las dificultades observadas es parte constitutiva de la tarea del docente. No se trata de enseñar competencias lexicogramaticales para ser aplicadas en la escritura, sino, por el contrario, identificar las dificultades de la escritura para provocar la tematización sobre esas competencias que es necesario desarrollar. No se propone enseñar una lengua abstracta que luego será aplicada en la escritura de verdaderos textos sino de enseñar a resolver los problemas que se les plantean a estos escritores concretos en las producciones de textos específicos. Esto no excluye que, en un momento posterior, una sistematización sobre los saberes necesarios para resolver estos problemas pueda resultar pertinente, pero entonces, la enseñanza cobrará el sentido de una herramienta para resolver un problema ya significado en la práctica de escribir.

\section{Bibliografía}

BRONCKART, J. P.; SCHNEUWLY, B.: La didactique du francais langue maternelle:

l'émergence d'une utopie indispensable.(s/d)

FERREIRO, E; PONTECORVO, C.; RIBEIRO MOREIRA, N.; GARCÍA HIDALGO, I. (1996):

Caperucita Roja aprende a escribir. Barcelona. Gedisa..

HICKS, D. (1996): Discourse, learning and schooling. USA. Cambridge University Press.

KAUFMAN, A. M.; RODRÍGUEZ, M. E. (1993): La escuela y los textos. Buenos Aires. Santillana.

LERNER, D. y otros (1995): Actualización curricular. Área Lengua. Dirección de Currículum. Secretaría de Educación. Municipalidad de la Ciudad de Buenos Aires.

La Nación (1997): Manual de estilo y ética periodística.

SCHUBAUER LEONI, M. L.; GROSSEN, M.: Negotiating the meaning of questions in didactic and experimental contracts. European Journal of Psychology of Education, III, 4, 451-471.

TOLCHINSKY, L. (1993): Aprendizaje del lenguaje escrito. España. Antrophos.

${ }^{1}$ El título de este artículo alude a una expresión que Antonio Tabucchi pone en boca del protagonista de su novela Sostiene Pereira: “...en los periódicos se escriben cosas que corresponden a la verdad o se asemejan a la verdad...".

${ }^{2}$ Trabajo realizado en el marco del proyecto de investigación "Revisión e Intercambio" del programa de Incentivos a la Investigación de la Secretaría de Políticas Universitarias del Ministerio de Educación de la Nación. Argentina.

${ }^{3}$ Prof. Titular cátedra Didáctica Primaria y Observación. Facultad de Humanidades y Ciencias de la Educación. Universidad Nacional de La Plata. Argentina.

${ }^{4}$ Auxiliar docente de la cátedra Didáctica Primaria y Observación. Facultad de Humanidades y Ciencias de la Educación. Universidad Nacional de La Plata. Argentina. 
${ }^{5}$ Las diferencias de edad se deben a repetición.

${ }^{6}$ Proyecto de la Secretaría de Extensión Universitaria de la Facultad de Humanidades y Ciencias de la Educación de la Universidad Nacional de La Plata- Argentina. Financiado por la Fundación W. K. Kellogg.

${ }^{7}$ El enunciado es una unidad operativa de análisis, de carácter gramatical, diferente al tipo de enunciación (de carácter pragmático) que también puede ser analizada en los textos, pero en cuyo análisis no se centrará este trabajo.

${ }^{8}$ Las transcripciones conservan los errores ortográficos de los alumnos.

${ }^{9}$ Las escrituras tachadas son supresiones y las subrayadas, agregados.

${ }^{10}$ La marca \# indica punto aparte en la producción del niño. 